\title{
Delay-Free Tracking Differentiator Design Based on Variational Mode Decomposition: Application on MEMS Gyroscope Denoising
}

\author{
Xi Zhang, ${ }^{1,2}$ Huiliang Cao $\mathbb{D}^{1,3}$ Chenguang Wang, ${ }^{1,3}$ Zhiwei Kou, ${ }^{1,3}$ Xingling Shao, \\ Jie Li, ${ }^{1,3}$ Jun Liu, ${ }^{1,3}$ and Chong Shen ${ }^{1,3}$ \\ ${ }^{1}$ Key Laboratory of Instrumentation Science \& Dynamic Measurement, Ministry of Education, North University of China, \\ Taiyuan 030051, China \\ ${ }^{2}$ School of Electrical and Control Engineering, North University of China, Taiyuan 030051, China \\ ${ }^{3}$ School of Instrument and Electronics, North University of China, Taiyuan 030051, China \\ Correspondence should be addressed to Chong Shen; shenchong@nuc.edu.cn
}

Received 25 October 2018; Accepted 3 March 2019; Published 14 April 2019

Academic Editor: Andrea Cusano

Copyright (C) 2019 Xi Zhang et al. This is an open access article distributed under the Creative Commons Attribution License, which permits unrestricted use, distribution, and reproduction in any medium, provided the original work is properly cited.

\begin{abstract}
This paper presents a delay-free tracking differentiator based on variational mode decomposition (VMD) for extracting the useful signal from a noisy measurement of gyroscope. Sigmoid function-based tracking differentiator (STD) is a novel tracking differentiator with the advantages of noise-attenuation ability and dynamical performance. However, there is a contradiction in STD; i.e., selecting a larger acceleration factor may cause faster convergence but bad random noise reduction whereas selecting a smaller acceleration factor may lead to signal delay but effective random noise reduction. Here, multiscale transformation is introduced to overcome the contradiction of STD. VMD is selected to decompose the noisy signal into multiscale components, and the correlation coefficients between each component and original signal are calculated, then the component with biggest correlation coefficient is reserved and other components are filtered by the proposed adaptive STD algorithm based on the correlation coefficient of each component, and finally the denoising result is obtained after reconstruction. The prominent advantages of the proposed algorithm are as follows: (i) compared to traditional tracking differentiators, better noise suppression ability can be achieved with suppression of time delay; (ii) compared to other widely used denoising methods, a simpler structure but better denoising ability can be obtained.
\end{abstract}

\section{Introduction}

In the early of 1990s, MEMS gyroscope was introduced by the Draper Laboratory. Due to the extensive research and advancements in fabrication technologies and readout electronics, MEMS gyroscope's performance has been improved over the last twenty years [1]. For further MEMS gyroscope research, a main motivation is the promise of achieving even better performance that competes with conventional bulky and expensive counterparts, but at small size and low cost $[2,3]$.

In the application of MEMS gyroscope, the noise becomes the main bottleneck which degrades of the signal accuracy. Therefore, it is very important to study the denoising technique to improve the performance of MEMS gyroscope. Many literatures have been dedicating great effort to remove MEMS gyroscope noises. Multiscale transformation method is a widely used technique for gyroscope denoising. In the mentioned wavelet-based techniques $[4,5]$, the proposed method combines a multidimensional gray model (GM) and a wavelet compression technique based on a novel IFOG structure with quadrature demodulation for noise suppression and sensitivity enhancement. In addition, multiscale transformation methods can decompose signal from timedomain into frequency-domain, and the high frequency noise can be eliminated for gyroscope denoising. In mode decomposition-based techniques [6-8], a hybrid algorithm of an optimized local mean decomposition-kernel principal 
component analysis (OLMD-KPCA) method is proposed for mechanism structure improvement. The optimal estimation theory is another widely used technique for gyroscope denoising, in which Kalman filter $(\mathrm{KF})$ is the most representative algorithm $[9,10]$. Besides, to solve the divergent problem of KF, adaptive Kalman filters (AKF) have been proposed [11-13], such as adaptive sampling strong tracking algorithm (ASSTA), residual based adaptive estimation (RAE), and multiple models based adaptive estimation (MMAE). Furthermore, AKF and variant one are applied to minimize the random noise in interferometric fiber-optic gyroscope (IFOG). ASSTA and scaled unscented Kalman filter (SUKF) algorithm are integrated for denoising the IFOG signal. Experimental results in literatures show that the adjusting $\mathrm{KF}$ or AKF mentioned before are suitable for gyroscope denoising in both static and dynamic conditions. However, the specific parameters in systems for the real-time filtering application still need to be improved. Moreover, many other denoising methods have been reported for gyroscope, such as forward linear predication (FLP) [14], time-frequency peak filtering (TFPF) [15], and hybrid denoising algorithms which can describe that an adaptive multiscale method based on the combination generalized morphological filter (CGMF) is presented for denoising [16]. These algorithms are available for gyroscope denoising but still with the problems of complex model or time delay.

In this paper, to develop a novel denoising algorithm for MEMS gyroscope, a Sigmoid function-based tracking differentiator (STD) based on multiscale decomposition is proposed with advantages of strong noise suppression ability and delay-free. In addition, through both simulations and experiments, the superiority and effectiveness of the proposed denoising algorithm in significantly reducing the noise are verified.

\section{Problem Formulation and Innovation Solution}

2.1. Sigmoid Function-Based TD. In order to provide the accurate estimation of derivative of virtual control, STD was first developed with the advantages of being simple structure, global fast convergence, and chattering-free in differential estimation. Here only a brief introduction of STD will be given. In [18], the detailed description can be found.

Lemma 1 (see [19]). The following system is considered:

$$
\begin{aligned}
\dot{z}_{1}(t) & =z_{2}(t) \\
\dot{z}_{2}(t) & =-\operatorname{sig}\left(z_{1}(t) ; a_{1}, b_{1}\right)-\operatorname{sig}\left(z_{2}(t) ; a_{2}, b_{2}\right) \\
\operatorname{sig}(x ; a, b) & =a\left[\left(1+e^{-b x}\right)^{-1}-0.5\right]
\end{aligned}
$$

where $\operatorname{sig}(\bullet)$ stands for the sigmoid function. If there exist $a_{1}>$ $0, a_{2}>0, b_{1}>0, b_{2}>0$, then the system is considered as globally asymptotically stable.
Lemma 2 (see [18]). The following novel tracking differentiator is considered:

$$
\begin{aligned}
& \dot{x}_{1}(t)=x_{2}(t) \\
& \dot{x}_{2}(t)=-R^{2}\left[\operatorname{sig}\left(x_{1}(t)-v(t) ; a_{1}, b_{1}\right)\right. \\
& \left.\quad+\operatorname{sig}\left(\frac{x_{2}(t)}{R} ; a_{2}, b_{2}\right)\right]
\end{aligned}
$$

where $R$ is referred to as acceleration factor and $R>0$. If there exist $a_{1}>0, a_{2}>0, b_{1}>0, b_{2}>0$, then for any arbitrary bounded and integral function $v(t)$ and a constant $T>0$, the solution of (2) satisfies

$$
\lim _{R \rightarrow \infty} \int_{0}^{T}\left|x_{1}(t)-v(t)\right| d t=0
$$

It can be concluded that $x_{1}(t)$, which is the tracking estimation, averagely converges to the input signal $v(t)$ and $x_{2}(t)$, which is the differential estimation, converges to the generalized derivative of $v(t)$.

2.2. Problem Formulation and Analysis. Although STD has already shown advantages of dynamical performance and noise-attenuation ability, note that STD is still constrained in supplying a relatively smooth denoising results without signal delay. This statement motivates us to pursue for an improved algorithm model which can radically relax the contradictory and give a better solution in suppressing noises. Here, a simulation noisy signal $Z(t)=\sin (2 \pi t / 3600)+\sin (2 \pi t / 60)+$ $\varphi(\mathrm{t})$ is utilized to depict the influence of acceleration factor $\mathrm{R}$ on the denoising performance of STD, where $\varphi(t)$ is random white noise. Figure 1 is the denoising results by using STD with different acceleration factor $R$, in which we can see that bigger $R$ means smoother denoising result but severer time delay, while smaller $R$ means worse denoising result but weaker signal delay. Therefore, it is really hard to select an exact R for STD just by experience to guarantee both smoother denoising result and smaller time delay especially for strong random noise.

In Figure 1, we know that noisy signal $Z(t)$ is composed of three components, which are $\sin (2 \pi \mathrm{t} / 3600), \sin (2 \pi \mathrm{t} / 60)$, and $\varphi(t)$, respectively. When STD is employed for filtering the noisy signal $\mathrm{Z}(\mathrm{t})$, it can be considered that STD is worked on the three components. The results of STD applied on the three components as are depicted in Figure 2, Figures 2(a), 2(c), and $2(\mathrm{e})$ are components $\sin (2 \pi \mathrm{t} / 3600), \sin (2 \pi \mathrm{t} / 60)$, and $\varphi(\mathrm{t})$. Figures 2(b), 2(d), and 2(f) are the filtered components by STD where the acceleration factor $R$ is set as 0.5 . From Figure 1(f) we can see that a favourable noise component suppression result is obtained. But in Figures 1(b) and 1(d), it can be seen that the time delay has occurred. Actually, the two components in Figures 2(a) and 2(c) do not need to be filtered, or in other words the STD with $R=0.5$ is inappropriate for filtering the two components, which leads to time delay. In traditional application, this phenomenon 


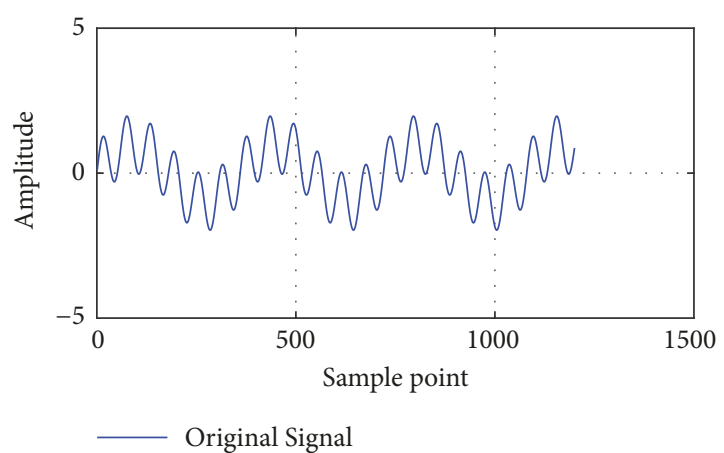

(a)

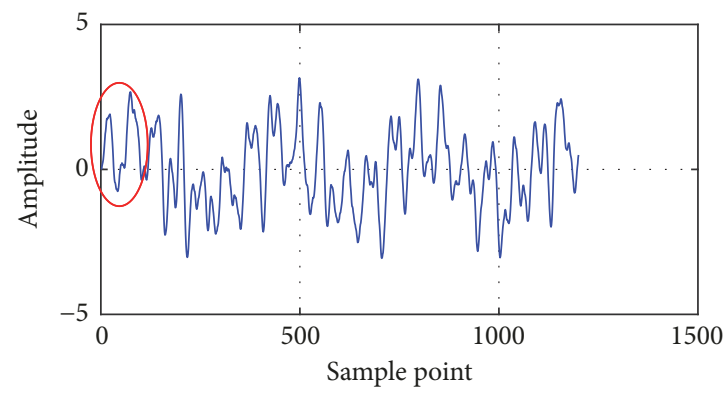

STD, $\mathrm{R}=2$

(c)

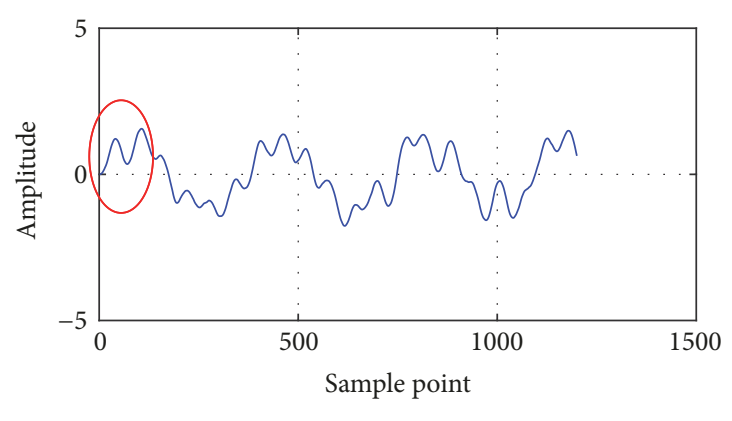

STD, $\mathrm{R}=0.5$

(e)

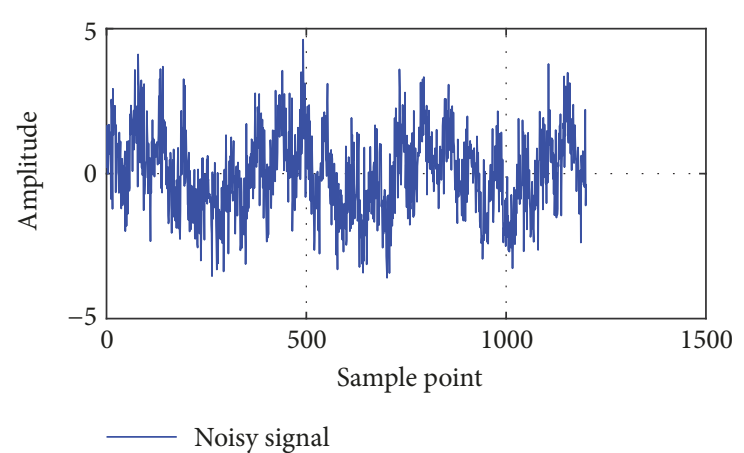

(b)

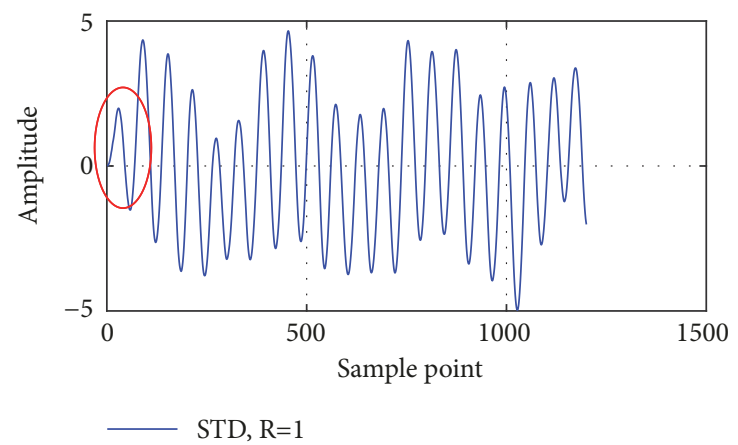

(d)

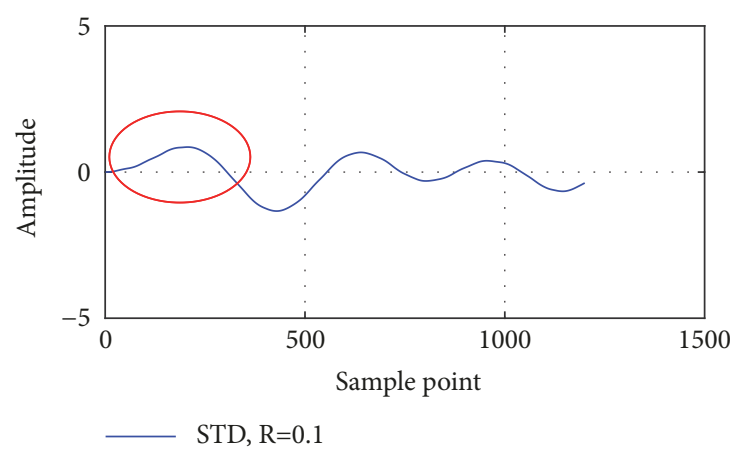

(f)

FIGURE 1: Denoising results of STD with different $R$.

happens inevitably due to the fact that the components are filtered by STD integrity as one signal. If we can decompose noisy signal into different components with different filtering ways by STD, the time delay phenomenon is expected to be restrained.

2.3. Innovation Solution. After analysis, how to decompose the noisy signal into different component and filter the components with different but appropriate acceleration factors become the key problem that needs to be resolved. As a novel nonrecursive signal processing technique, VMD can adaptively decompose a real valued signal into discrete set of band-limited subsignals intrinsic mode functions (BLIMFs) owing specific sparsity properties [20]. Thus, in our study, VMD is selected for decomposing noisy signal before the application of STD.
There is another important issue that needs to be resolved, which is how to determine the acceleration factor $(R)$ of STD for each BLIMF after VMD decomposition. The traditional method is determining $R$ just by experience; however, the denoising effect cannot be guaranteed. The correlation coefficient $(C C)$ can be used to reflect the correlation degree between BLIMF and original signal. Hence, $C C$ is used here as a determination criterion for each $R$ of STD. The steps of VMD-STD algorithm are given below [21].

Step 1 (decomposition). The VMD is utilized to decompose the signal into BLIMFs, which are $u_{1}, u_{2} \ldots u_{n}$, respectively.

VMD is a novel method of signal decomposition; multicomponent signals are decomposed into BLIMFs, so as to minimize the sum of the bandwidth estimation of each 


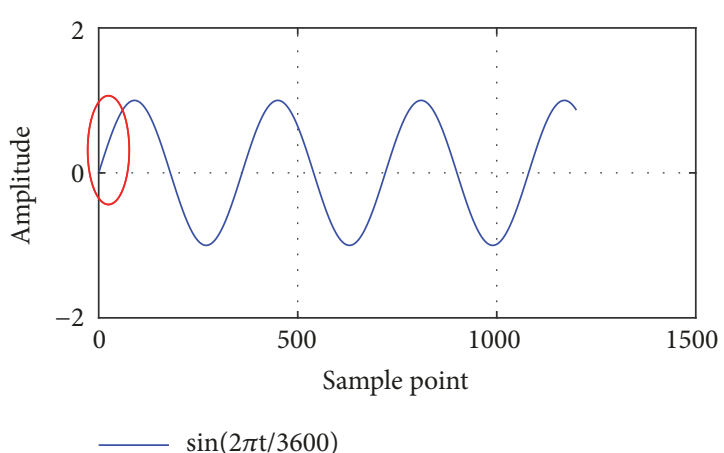

(a)

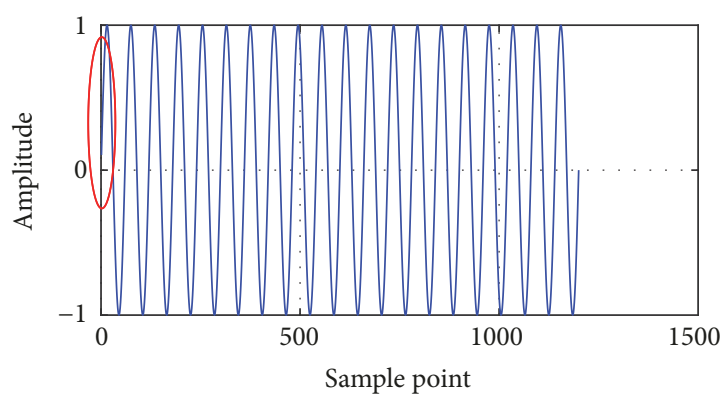

$-\sin (2 \pi \mathrm{t} / 60)$

(c)

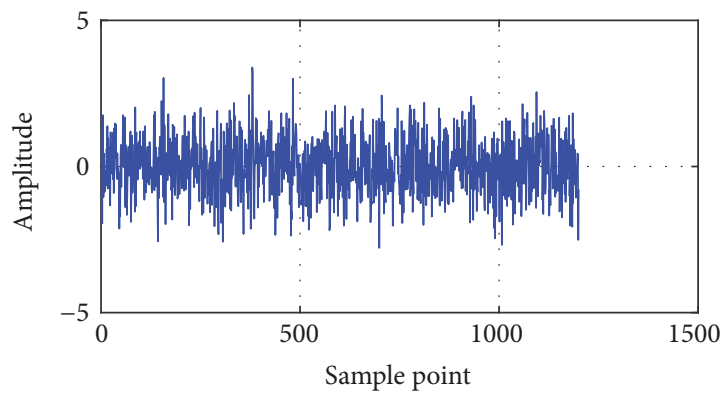

White noise

(e)

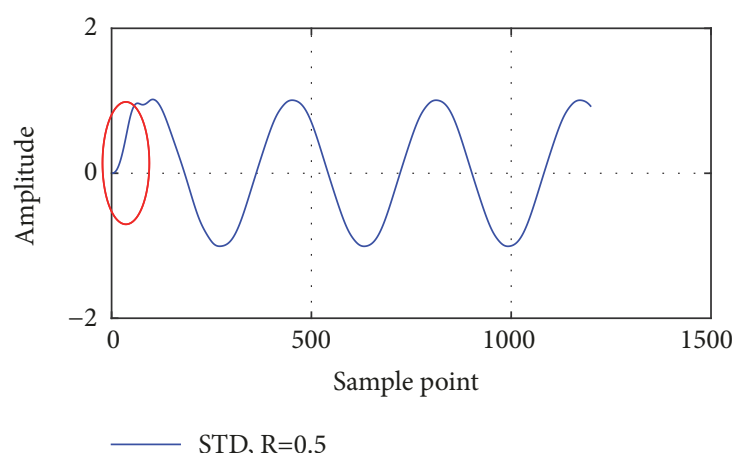

(b)

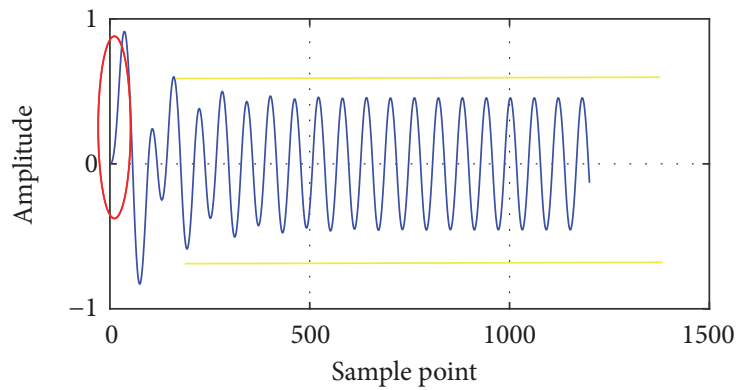

STD, $\mathrm{R}=0.5$

(d)

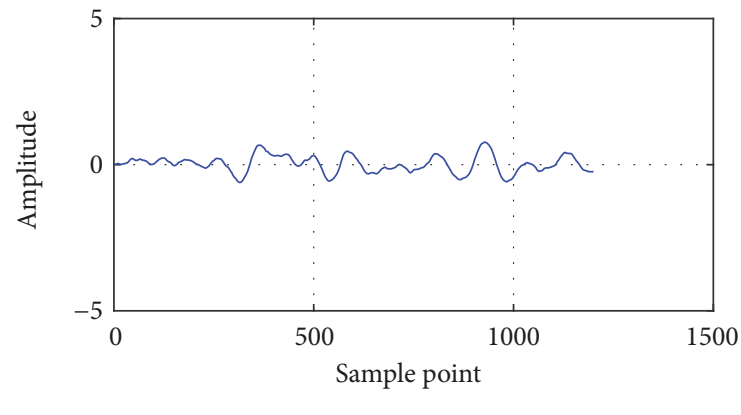

STD, $\mathrm{R}=0.5$

(f)

FIgURE 2: The results of STD applied on the three components.

mode. VMD can be represented as a constrained variational problem which is given by

$$
\begin{aligned}
& \min _{\left\{u_{k}\right\},\left\{\omega_{k}\right\}}\left\{\sum_{k}\left\|\partial_{t}\left[\left(\delta(t)+\frac{j}{\pi t}\right) * u_{k}(t)\right] e^{-j \omega_{k} t}\right\|_{2}^{2}\right\} \\
& \text { s.t. } \quad \sum_{k} u_{k}=Z(t)
\end{aligned}
$$

where $u_{k}$ is the $k$ th mode, $Z(t)$ is the original signal to be decomposed, $\omega_{k}$ is the corresponding center frequency, $\vartheta_{t}$ represents gradient with respect to $t$, and $\delta(\mathrm{t})$ is the impulse function.
Step 2. Calculate $C C$ for each BLIMF, where $m$ is the length of $Z(t)$.

$$
\begin{array}{r}
C C_{j}=\frac{\sum_{i=1}^{m}\left(z_{i}-\bar{z}\right)\left(u_{j i}-\bar{u}_{j}\right)}{\sqrt{\left[\sum_{i=1}^{m}\left(z_{i}-\bar{z}\right)^{2} \sum_{i=1}^{m}\left(u_{j i}-\bar{u}_{j}\right)^{2}\right]}} \\
\quad i=1,2, \ldots m ; j=1,2, \ldots n .
\end{array}
$$

Step 3. Judge the biggest $C C_{j}$ and select the corresponding $u_{j}$. Set an origin value of $R$; then STD is used to track $u_{j}$ and the tracking result $d_{-} u_{j}$ is obtained. Calculate the difference between $u_{j}$ and $d_{-} u_{j}$; if the difference is smaller than the defined threshold $\theta$, then $R$ is selected as $R_{j}$; if the difference is bigger than $\theta$, adjust the value of $R$ until $u_{j}-d_{-} u_{j} \leq \theta$ 


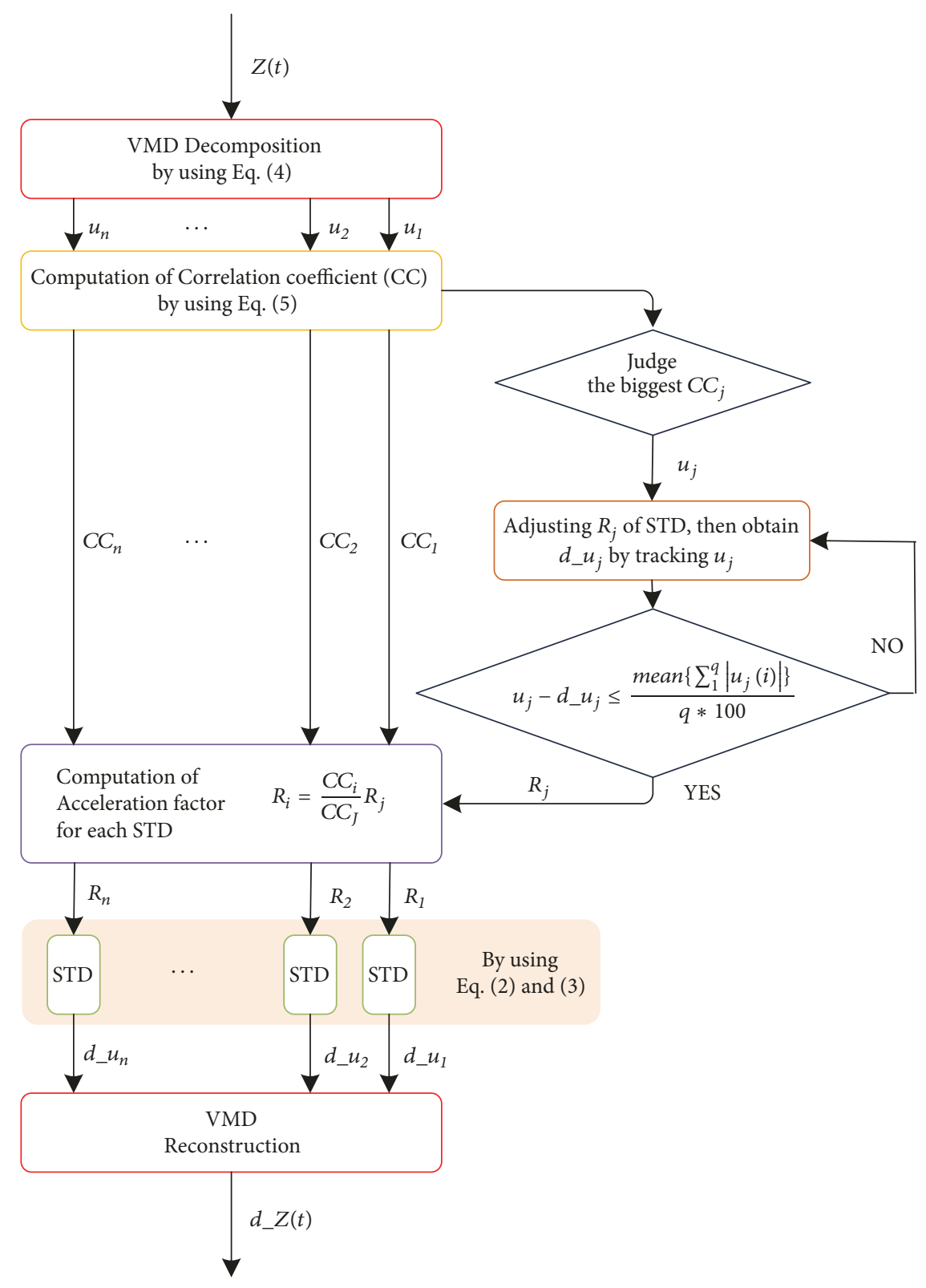

FIGURE 3: Flowchart of VMD-STD algorithm.

is reached. The threshold $\theta$ is defined as (6) by experience, where $q$ is the length of $u_{j}$.

$$
\theta=\frac{\operatorname{mean}\left\{\sum_{1}^{q}\left|u_{j}(i)\right|\right\}}{q * 100}
$$

After $R_{j}$ is determined, the other $R_{i}$ can be calculated by (7), where $i=1,2, \ldots, n, i \neq j$.

$$
R_{i}=\frac{C C_{i}}{C C_{j}} R_{j}
$$

Step 4. Design $n$ STDs for all the BLIMFs by using the calculated $R$ in (7). Then each BLIMF is denoised by the corresponding STD.

Step 5 (reconstruction). The last step is to get the final denoising result by reconstruction. Note that $u_{j}$ with the biggest $C C_{j}$ is the most useful component of original signal according to the definition of correlation coefficient; therefore $u_{j}$ will be reserved and this step can make sure that the time-delay would be avoid. The $d_{\_} u_{1}, d_{-} u_{2}, \ldots, d_{-} u_{j}, \ldots, d_{-} u_{n}$ are added together and the final denoising result is obtained.

Since the proposed denoising algorithm is the combination of STD and VMD, it is named as VMD-STD algorithm. The flowchart of VMD-STD algorithm is shown as Figure 3. 


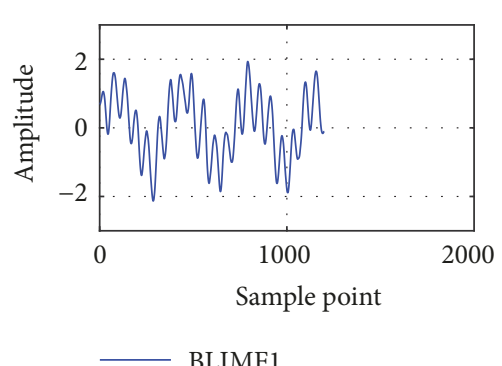

(a)

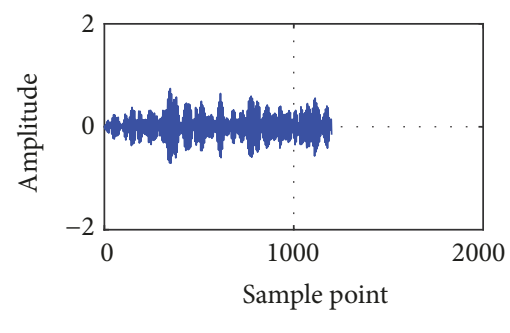

BLIMF4

(d)

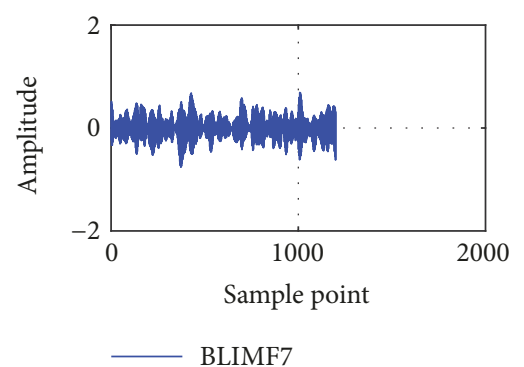

(g)

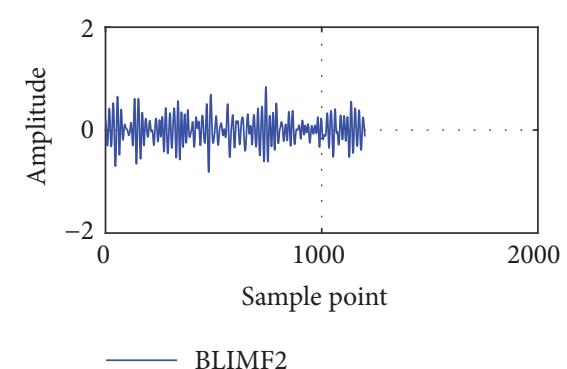

(b)

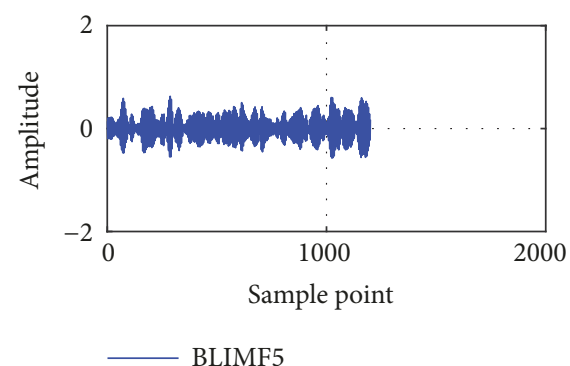

(e)

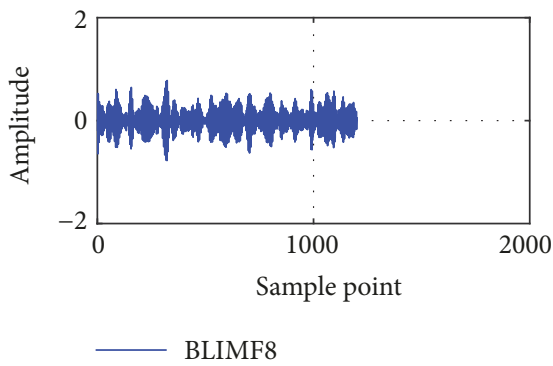

(h)

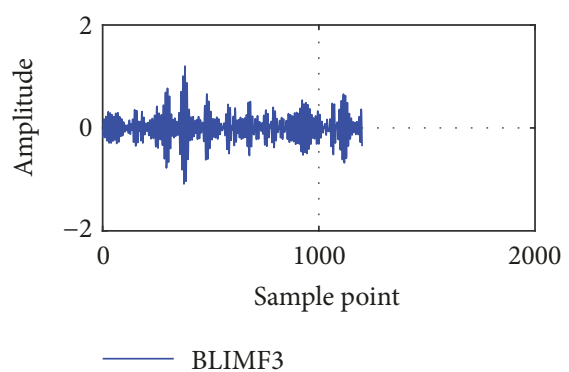

(c)

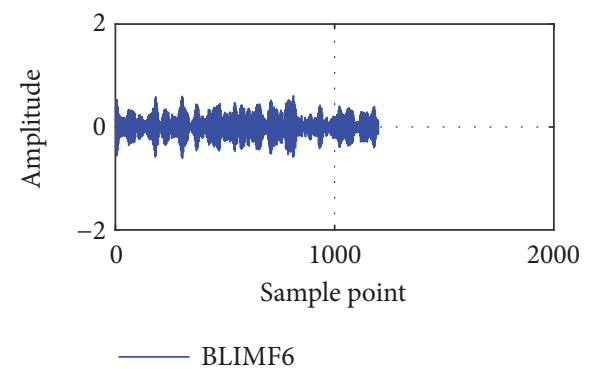

(f)

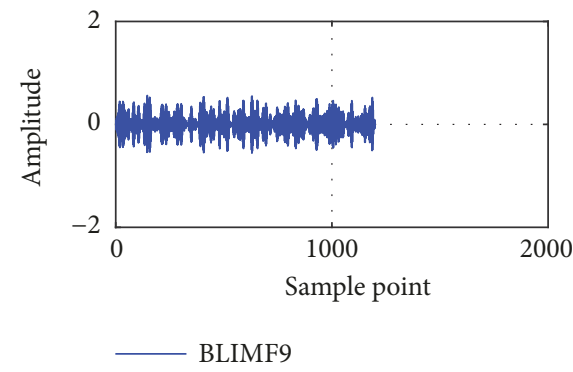

(i)

FIGURE 4: Decomposition results of $Z(t)$ by VMD.

\section{Simulation}

3.1. Simulated Signal Process. In order to present the superiority of the proposed VMD-STD denoising algorithm, the simulated signal $Z(t)$ is employed for verification. According to the steps of VMD-STD algorithm, firstly $Z(t)$ is decomposed into BLIMFs as shown in Figure 4. From Figure 4 it can be seen that low frequency components are extracted mainly in BLIMF1 and high frequency noise components are mainly in other BLIMFs. Therefore, STDs for each BLIMF should be designed separately.

The decomposition is the first step of the proposed VMDSTD algorithm, and the second step is to calculate the CC of each BLIMF. The calculation results are shown in Table 1 , from where it can be seen that BLIMF1 has the biggest CC. The third step is applying STD on BLIMF1 to determine the first $R$ according to criterion in Step 3, and the other acceleration factors $R$ can be obtained by (5).

The next step is the application of STD on BLIMFs with determined $R$. It is noted that BLIMF1 will be reserved without any processing, which can guarantee there is no signal delay of low frequency component. Figure 5 is the denoising results of each BLIMF by using STD with determined $R$. It can be seen that the details of BLIMF1 are reserved totally; BLIMF2 is filtered by STD with $R=0.36$ which makes part of the details reserved, and signal delay is occurring compared to Figure 4(b); BLIMF3 to BLIMF9 are filtered by STD with bigger $R$ which make a set of smooth denoising results obtained, and it goes without saying that the signal delays are occurring.

The last step is reconstruction. All the filtered components in Figure 5 are added together and then the reconstruction result is obtained, which is shown as Figure 6(c). Comparison between Figures 6(b) and 6(c) show that the noise is filtered effectively; comparison between Figures 6(a) and 6(c) show that there is almost no signal delay occurring after denoising by the proposed VMD-STD algorithm due to the fact that the low frequency component BLIMF1 is reserved directly; from Figure 6(d) we can see that the residual is still existing, which means the noise-attenuation ability or signal distortion problem of STD still needs to be considered which would be our future work.

But beyond that, the prior work conducts a simulation process with the single VMD-STD algorithm. To verify 


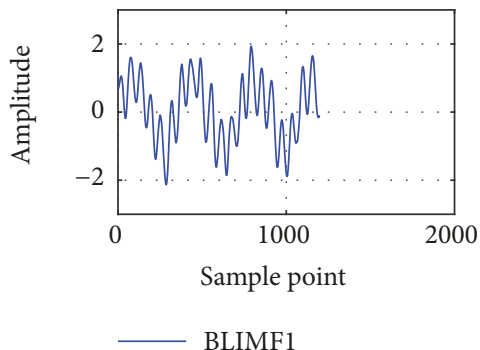

(a)

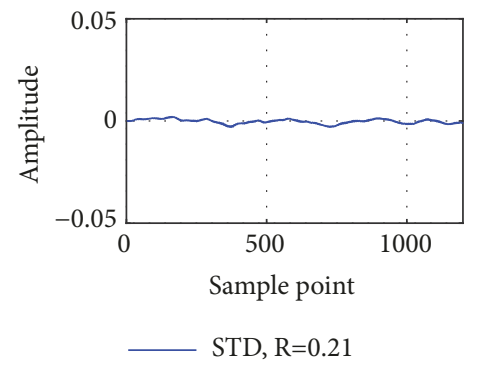

(d)

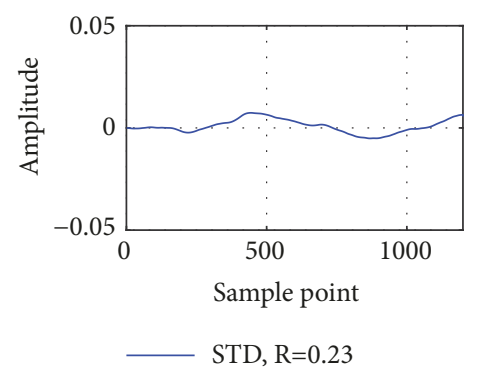

(g)

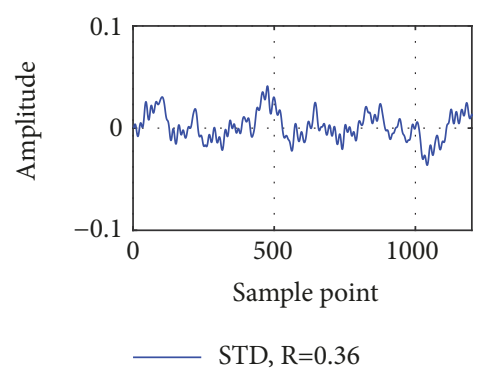

(b)

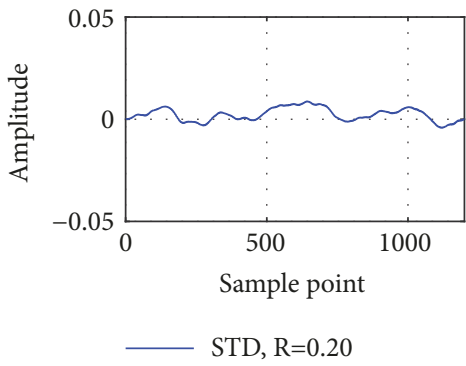

(e)

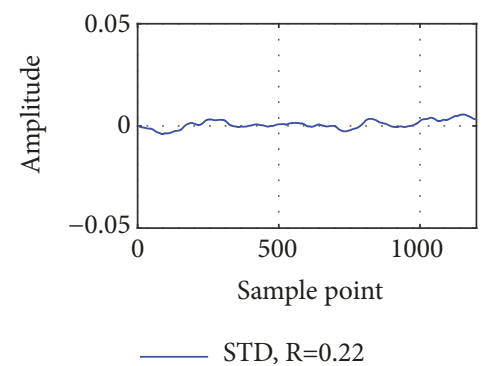

(h)

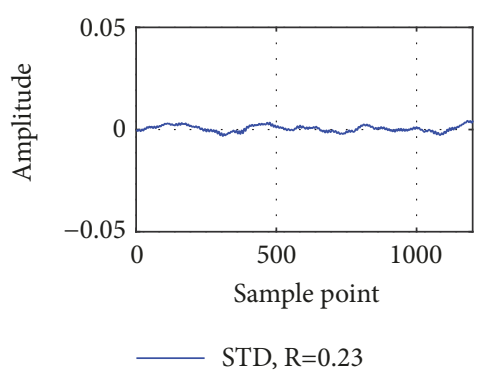

(c)

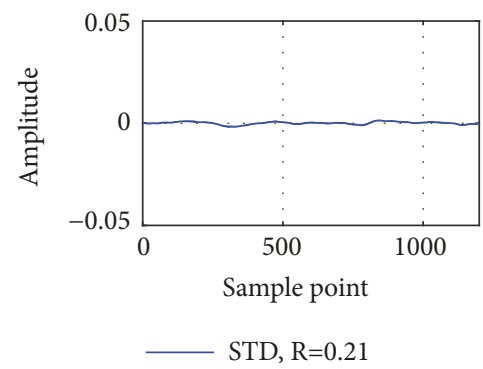

(f)

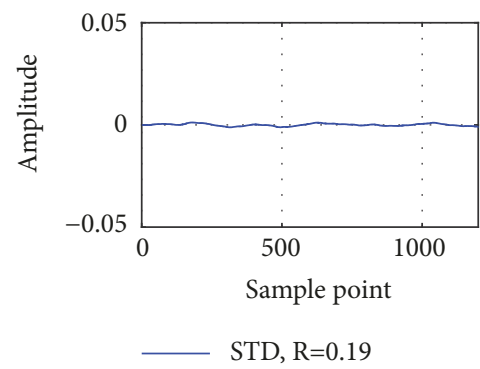

(i)

FIGURE 5: Denoising results of each BLIMFs by using STD with determined R.

TABLE 1: The correlation coefficients of BLIMFs in Figure 4.

\begin{tabular}{lccccccccc}
\hline & BLIMF1 & BLIMF2 & BLIMF3 & BLIMF4 & BLIMF5 & BLIMF6 & BLIMF7 & BLIMF8 & BLIMF9 \\
\hline Correlation coefficient (CC) & 0.75 & 0.32 & 0.21 & 0.20 & 0.19 & 0.20 & 0.21 & 0.20 & 0.18 \\
\hline Acceleration factor (R) & 0.8 & 0.36 & 0.23 & 0.21 & 0.20 & 0.21 & 0.23 & 0.22 & 0.19 \\
\hline
\end{tabular}

our proposed algorithm feasible indeed, some other advanced denoising methods are applied for comparison, like adaptive robust Kalman filter (ARKF), detrended fluctuation analysis-VMD (DFA-VMD), and empirical mode decomposition-forward linear prediction (EMD-FLP). These methods can be also well applied for noise suppression with gyroscope output signal. In Table 2, we added the corresponding noise of the different signal-to-noise power ratios (SNRs) to verify the noise reduction capability. Comparing DFA-VMD, EMD-FLP, VMD-STD, and ARKF methods, the DFA-VMD and EMD-FLP have similarity in function for noise reduction. The ARKF method has good suppression effect, but the SNR from denoising gyroscope signal of VMD-STD is relatively higher. Consequently, our proposed VMD-STD method is superior to other denoising algorithms.
3.2. Computational Complexity of VMD-STD Denoising Algorithm. In order to assess the complexity of the proposed VMD-STD algorithm, the computation time and space complexity are analysed. Firstly, we assume that the time cost of each operator is the same; therefore only the performance and running hardware are concerned. All required operations, such as addition (ADD), subtraction (SUB), multiplication (MUL), definition (DEF), and division (DIV) are considered. $S$ is introduced to stand for the length of input signal, i.e., the data scale, and $N$ is introduced to stand for the maximum of loops and iterations. The VMD algorithm has already been analysed by [20], as shown in Table 3 . The simulated signal in this paper is decomposed into $k$ modes, and the variate $\mathrm{N}$ in Table 2 stands for the maximum number of iterations.

In our VMD algorithm, the initialization parameters are set as follows: alpha $=2000$, tau $=0$, tol $=1 \mathrm{e}-7$, and $\mathrm{N}=$ 


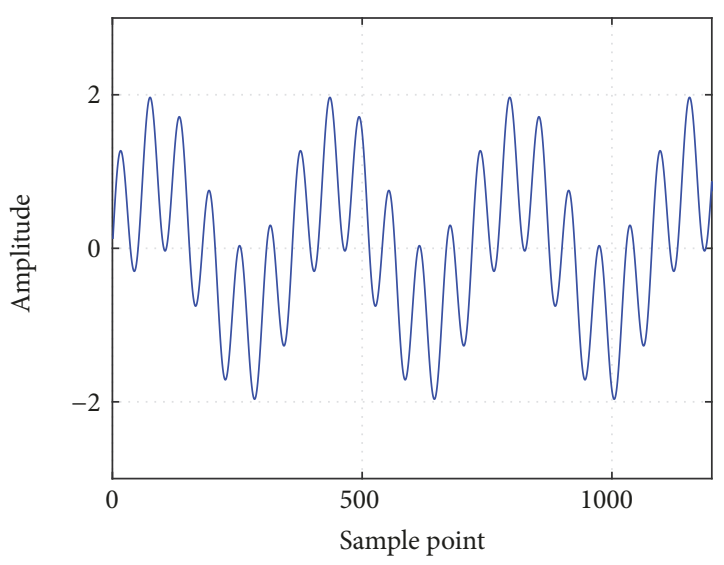

Original signal

(a)

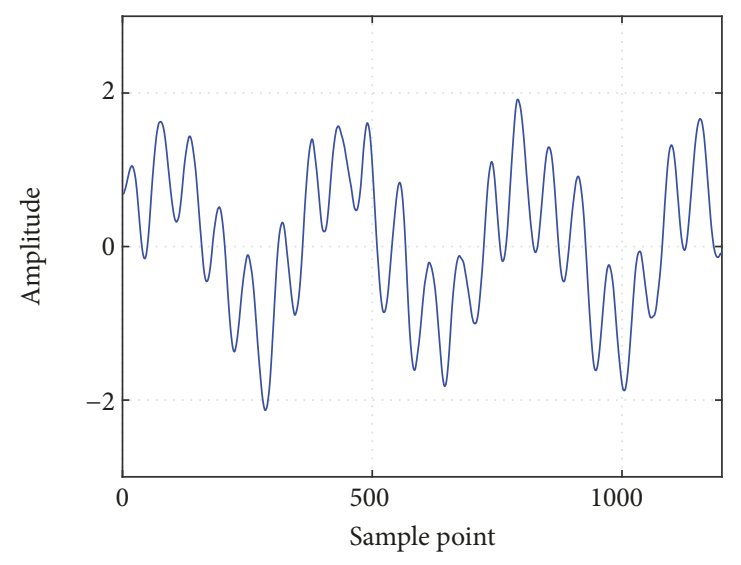

- De-noised signal

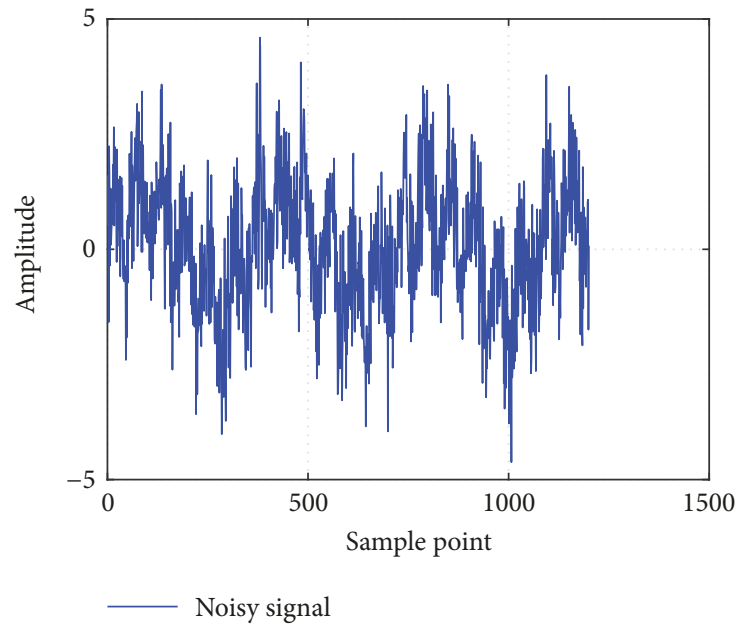

(b)

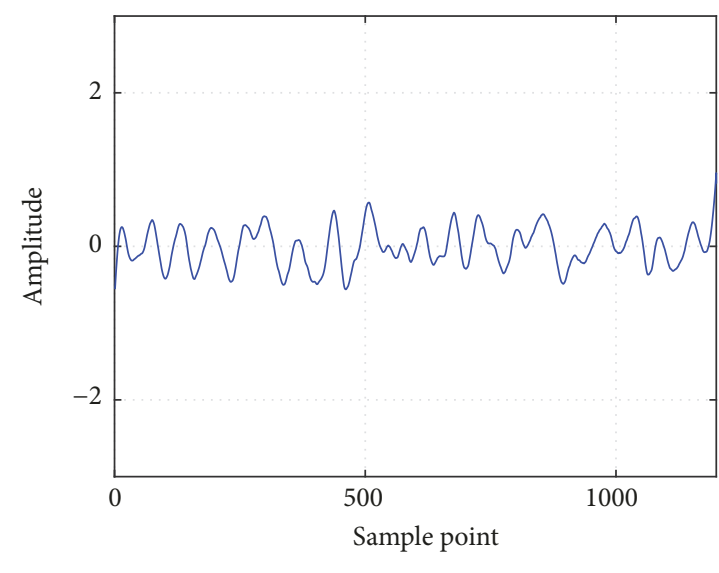

Residual

(c)

(d)

FIGURE 6: Reconstruction results and comparisons.

TABLE 2: Comparison of the VMD-STD with other methods with different SNRs.

\begin{tabular}{lccccccccccccc}
\hline SNR $(\mathrm{dB})$ & -20 & -16 & -9 & -3 & 0 & 3 & 6 & 9 & 11 & 13 & 16 & 20 \\
\hline EMD-FLP & -14.51 & -10.23 & -3.63 & 1.76 & 3.29 & 7.85 & 9.67 & 11.96 & 14.64 & 15.79 & 18.34 & 21.15 \\
DFA-VMD & -16.55 & -12.35 & -5.59 & 0.38 & 6.54 & 6.48 & 12.77 & 12.47 & 14.25 & 16.73 & 18.90 & 22.56 \\
ARKF & -12.71 & -9.21 & -0.41 & 4.69 & 7.12 & 10.30 & 13.49 & 15.68 & 17.73 & 19.59 & 21.30 & 24.87 \\
VMD-STD & -9.70 & -3.98 & 1.77 & 6.75 & 10.14 & 13.15 & 13.87 & 17.06 & 17.96 & 19.71 & 22.57 & 25.61 \\
\hline
\end{tabular}

TABLE 3: Evaluating time and space complexity for the VMD algorithm.

\begin{tabular}{lcc}
\hline function & $\mathrm{T}$ & $\mathrm{M}$ \\
\hline Initialize & $\mathrm{O}\left(2 \mathrm{~N} \log _{2}^{2 N}\right)$ & {$[(3+\mathrm{K} \cdot N+N) \cdot 2 \mathrm{~S}+\mathrm{K} \cdot N]$ float } \\
Update $\boldsymbol{u}_{\boldsymbol{k}}$ & $(6 \mathrm{ADD}+2 \mathrm{MUL}+2 \mathrm{DIV}) \cdot \mathrm{K} \cdot N \cdot 2 \mathrm{~S}$ & {$[2 \mathrm{~S}]$ float } \\
Update $\boldsymbol{w}_{\boldsymbol{k}}$ & $(2 \mathrm{CMP}+3 \mathrm{MUL}+2 \mathrm{ADD}) \cdot \mathrm{K} \cdot N \cdot \mathrm{S}$ & 0 \\
Dual ascent & $(4 \mathrm{ADD}+1 \mathrm{MUL}) \cdot N \cdot 2 \mathrm{~S}$ & 0 \\
Convergence & $(4 \mathrm{ADD}+2 \mathrm{MUL}) \cdot N \cdot 2 \mathrm{~S}$ & 0 \\
Complexity & $\mathrm{O}\left(2 \mathrm{~N} \log _{2}^{2 N}\right)$ & $\mathrm{O}(N)$ \\
\hline
\end{tabular}


TABLE 4: Time and space complexity for the STD based on the VMD algorithm.

\begin{tabular}{lcc}
\hline Function & $\mathrm{T}$ & $\mathrm{M}$ \\
\hline STD & $\mathrm{O}(N)$ & {$[\mathrm{S}+5 \mathrm{~N} \cdot \mathrm{S}+11]$ float } \\
VMD & $\mathrm{O}\left(2 \mathrm{~N} \log _{2}^{2 N}\right)$ & {$[(4+\mathrm{K} \cdot \mathrm{N}+\mathrm{N}) \cdot 2 \mathrm{~S}+\mathrm{K} \cdot \mathrm{N}]$ float } \\
STD based on VMD & $\mathrm{O}\left(2 \mathrm{~N} \log _{2}^{2 N}\right)+(\mathrm{K}+1) \cdot \mathrm{O}(N)+k^{\prime} \cdot 1$ ADDD $\mathrm{S}$ & {$[(4+\mathrm{K} \cdot N+N) \cdot 2 \mathrm{~S}+\mathrm{K} \cdot \mathrm{N}]+(\mathrm{K}+1) \cdot[\mathrm{S}+5 N \cdot \mathrm{S}+11]$ float } \\
Complexity & $\mathrm{O}\left(2 \mathrm{~N} \log _{2}^{2 N}\right)$ & $\mathrm{O}(N)$ \\
\hline
\end{tabular}

TABLE 5: Execution times for a simulated signal applying different noise suppression methods.

\begin{tabular}{lcccc}
\hline $\mathrm{n}$ & EMD-FLP & DFA-VMD & ARKF & VMD-STD \\
\hline $2^{8}$ & 0.1514 & 0.1847 & 0.2094 & 0.1643 \\
$2^{9}$ & 0.2632 & 0.3028 & 0.7036 & 0.2871 \\
$2^{10}$ & 0.4876 & 0.6178 & 1.0962 & 0.5974 \\
$2^{11}$ & 0.9247 & 1.3842 & 2.4621 & 1.3625 \\
$2^{12}$ & 1.2148 & 1.9634 & 2.8693 & 1.8712 \\
$2^{13}$ & 2.5642 & 3.5613 & 6.1679 & 3.2964 \\
$2^{14}$ & 5.0469 & 7.0951 & 15.1413 & 6.9451 \\
$2^{15}$ & 9.8346 & 16.2846 & 31.5973 & 12.8753 \\
$2^{16}$ & 22.3765 & 32.8897 & 66.8432 & 26.3154 \\
\hline
\end{tabular}

1000. In Table 3, the time and space complexity of VMD are of logarithmic order $\mathrm{O}\left(2 \mathrm{~N} \log _{2}^{2 N}\right)$ and linear order $\mathrm{O}(\mathrm{N})$, respectively.

In the STD algorithm, the latter relates specifically to the parameter initialization and system function output. From the detailed computation, the STD's time and space complexity are both of linear order $\mathrm{O}(\mathrm{N})$ and linear order $\mathrm{O}(\mathrm{N})$.

As shown in Table 4, the time and space complexities for the STD based on the VMD algorithm are listed. The time complexity is of logarithmic order $\mathrm{O}\left(2 \mathrm{~N} \log _{2}^{2 N}\right)$, whereas the space complexity is of linear order $\mathrm{O}(\mathrm{N})$. In general, we evaluate the magnitude of the complexity $\mathrm{O}\left(\mathrm{N}^{3}\right)>$ $\mathrm{O}\left(N^{2}\right)>\mathrm{O}\left(N \log _{2}^{N}\right)>\mathrm{O}(N)>\mathrm{O}\left(\log _{2}^{N}\right)>\mathrm{O}(\mathrm{C})$. Hence, the results of the analysis demonstrate VMD-STD method is a valid algorithm solvable in polynomial time.

To compare the execution time of different denoising algorithms, a simulation with signals of lengths ranging from $2^{8}$ to $2^{16}$ with $\mathrm{SNR}=5 \mathrm{~dB}$ was performed on a personal computer (Intel ${ }^{\circledR}$ Core $^{\mathrm{TM}} @ 2.80 \mathrm{GHz}$ and 8 GB RAM memory) running Windows 10.

Comparing these current algorithms with VMD-STD, the same experimental conditions were applied in the tests. The actual execution times are listed in Table 5. By complexity analysis, EMD-FLP time complexity is of $\mathrm{O}\left(2 \mathrm{~N} \log _{2}^{2 N}\right)$ and DFA-VMD is of $\mathrm{O}\left(2 \mathrm{~N} \log _{2}^{2 N}\right)$. The ARKF algorithm has the largest magnitude of $\mathrm{O}\left(\mathrm{N}^{3}\right)$. The STD-VMD time complexity is equal to DFA-VMD and EMD-FLP whereas the execution times indicate that VMD-STD increases more in time than DFA-VMD and less than EMD-FLP. It can be explained that the influences of algorithm factors are different. The actual VMD-STD algorithm is with a larger factor than FLP but smaller than DFA. Thus, the proposed algorithm has a simple and rational construction as a whole.

\section{Experimental and Verification}

4.1. Denoising Test. In this section, the output of gyroscope is employed for verifying the effectiveness of VMD-STD denoising algorithm. MEMS S-springs vibrating ring gyroscope (MSVRG) $[17,22]$ is employed in this paper, and the structure is shown in Figure 7; its mode analysis is shown in Figure 8.

As Figure 7 shows, a glass substrate with patterned electrode leads, a ring resonator, and twenty-four silicon capacitor electrodes which are evenly distributed around the ring are used to form the MSVRG. On the upper surface of the glass, the patterned metal leads connected to the silicon electrodes are provided to realize the electrical conduction with the measuring and controlling circuit of the gyroscope. In Figure 8, the resonant frequencies in the operating modes of the ring resonator are $8.8078 \mathrm{kHz}$ and $8.8050 \mathrm{kHz}$, respectively, and the frequency splitting value is $2.8 \mathrm{~Hz}$. Figure 9 is the experimental setup of gyroscope. The equipment is mainly included: temperature control cabinet, a single axis MSVRG, data acquisition system (Multimeter), computer, and Power Supply.

In this work, one set of data is collected from gyroscope with temperature changing from $+10^{\circ} \mathrm{C}$ to $-10^{\circ} \mathrm{C}$ to $+10^{\circ} \mathrm{C}$, where the temperature change rate is less than $1^{\circ} \mathrm{C} / \mathrm{min}$. From Figure 10 we can see that, during the temperature variation, there is an obvious drift trend which is submerged in large noises. To extract the precise drift, it is necessary to eliminate the noises effectively. The denoising procedure by using VMD-STD algorithm for gyroscope output signal is the same as section "Simulation." At the same time, we also apply other advanced denoising methods for signal denoising. ARKF, DFA-VMD, EMD-FLP, and traditional STD are employed for comparison as shown in Figure 10.

From Figure 10 it can be obviously seen that the noises of original signal are removed. However, the denoising effects are different among different denoising algorithms. When STD algorithm is employed for denoising, a smooth denoising result can be obtained but the signal delay is happening. When DFA-VMD, EMD-FLP, ARKF, and VMDSTD algorithms are employed for denoising, the signal delay is avoided, and more effective denoising results are obtained. From the principle of DFA-AMD we can see that the relevant modes composed of VMD can be selected by DFA and then used for the construction of denoised signal, which means that the BLIMFs with smaller DFAs would 


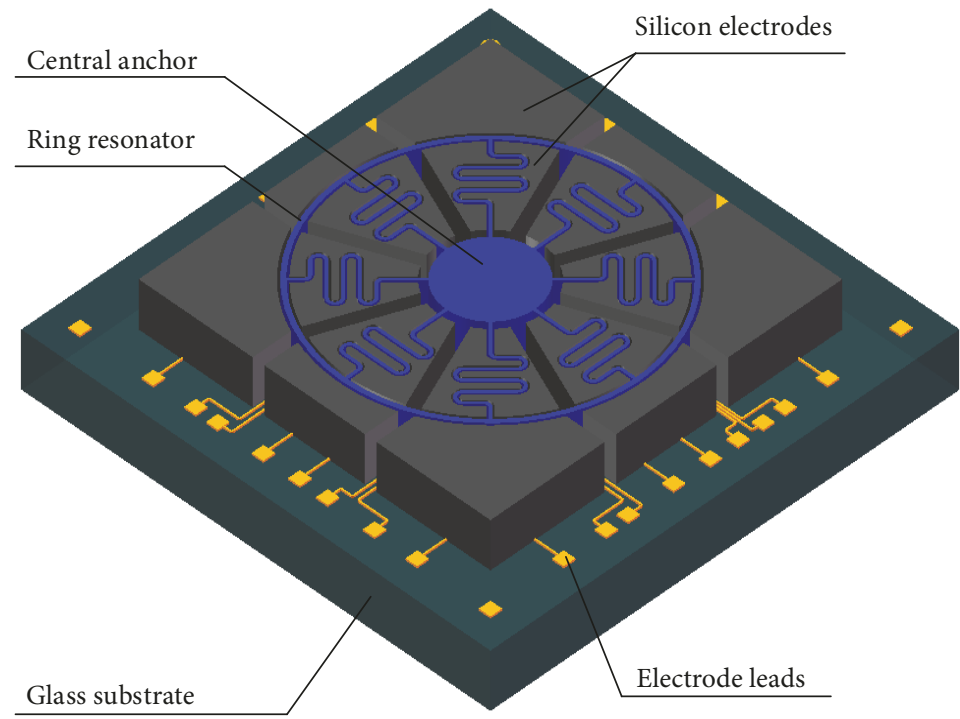

(a)

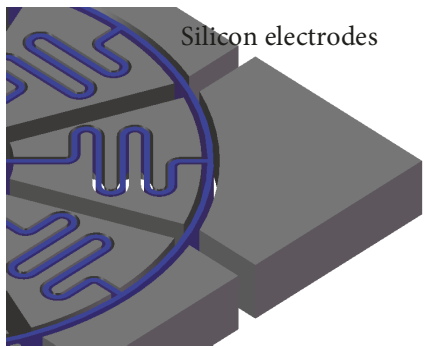

(b)

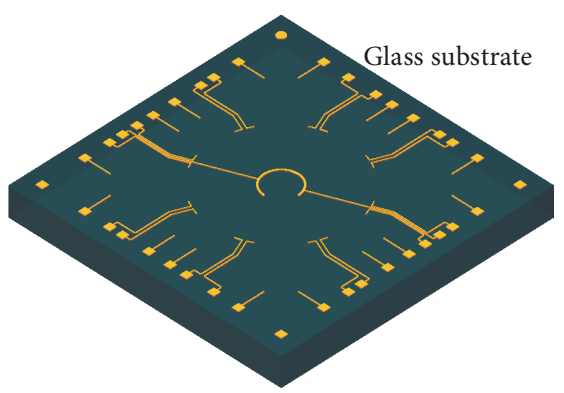

(c)

Figure 7: Schematic of the MSVRG. (a) Whole structure of MSVRG; (b) silicon capacitor electrodes; (c) the glass substrate with patterned electrode leads [17].

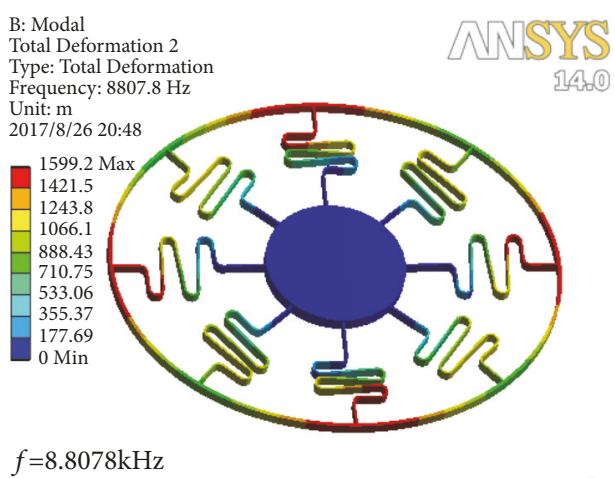

(a)

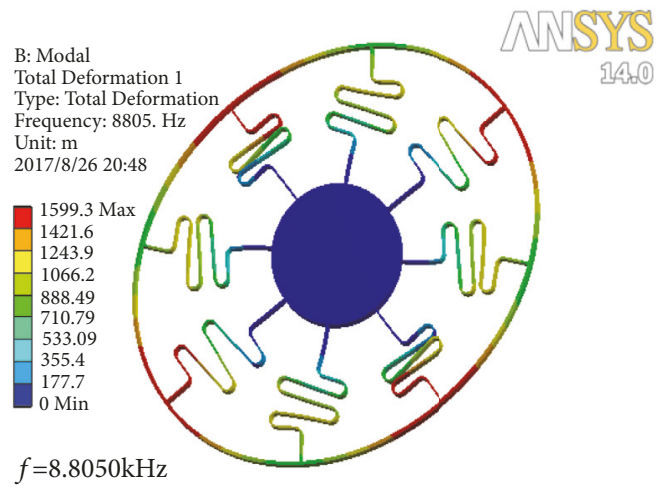

(b)

Figure 8: (a) Drive mode shapes and frequencies of ring resonator; (b) sensitive mode shapes and frequencies of ring resonator [17].

be abandon, and the rest of BLIMFs are remaining for reconstruction; therefore the noise in the remaining BLIMFs will be reserved. While in the process of VMD-STD, almost all the BLIMFs would be processed by STD except the BLIMF with biggest correlation coefficient, and the smaller correlated BLIMFs would be suppressed strictly; therefore the denoising performance of VMD-STD algorithm is better. The principle of EMD-FLP algorithm is decomposing the signal into IMFs by EMD and then filtering each IMF by FLP, which is similar as our proposed VMD-STD algorithm. However, there is no selection criterion for the parameters of FLP for each IMF which means that it is hard to select the best parameters 


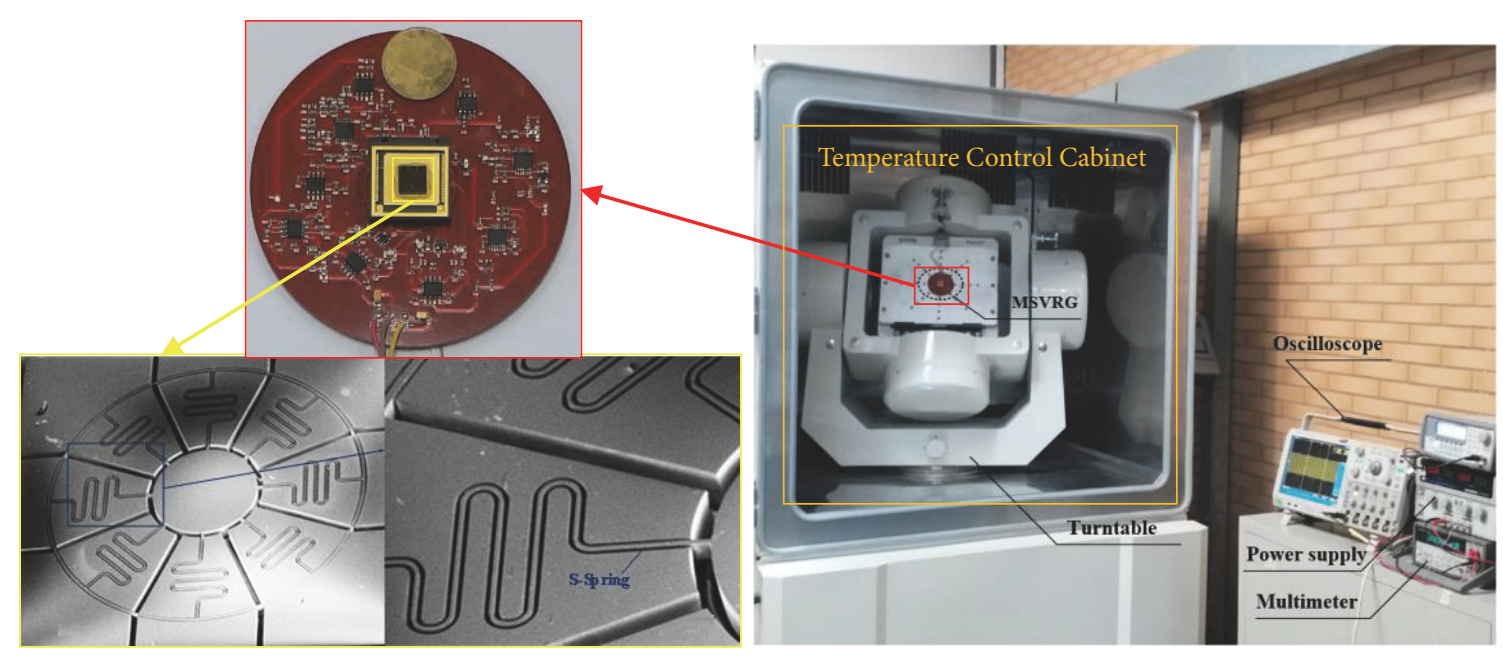

FIGURE 9: Experimental equipment arrangement of MSVRG.

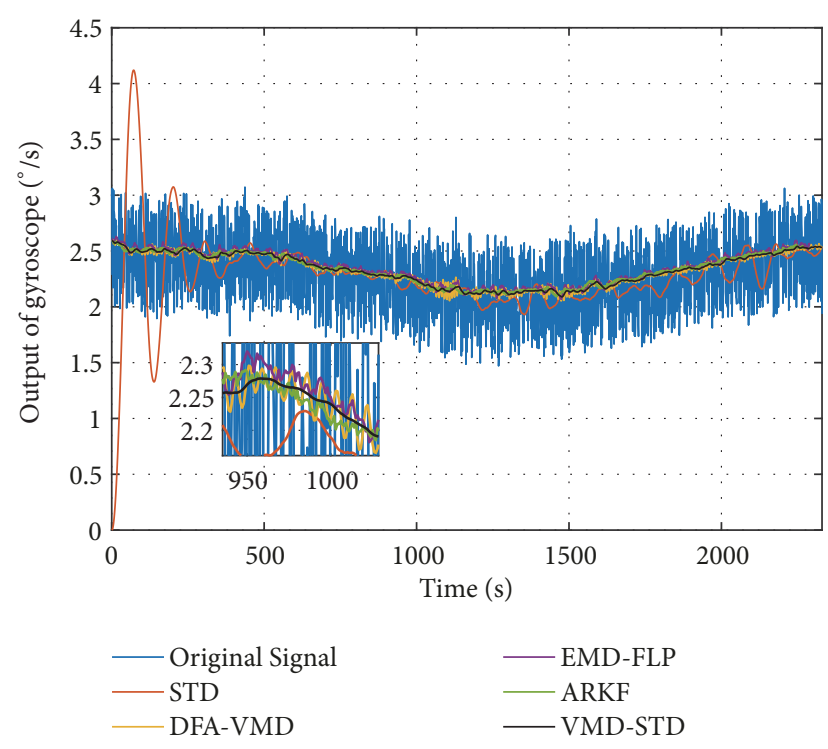

FIgURE 10: Comparison results of different denoising methods.

just by experience; therefore the denoising result of EMDFLP algorithm is not optimal. The denoising result of ARKF is not as smooth as VMD-STD algorithm; that is because ARKF is denoising the signal in time-domain directly, while the VMD-STD algorithm is decomposing the time-domain signal into different BLIMFs firstly and then each BLIMF is denoised independently which makes the denoising process more specific. Besides, compared to ARKF, it does not need to predesign any model in the application of VMD-STD, which makes the structure of the proposed algorithm simpler.

To evaluate the denoising ability of the proposed algorithm, Allan variance analysis is introduced to quantitative comparison. By using Allan variance analysis, the noise coefficients of MEMS gyroscope can be identified and evaluated. Normally, the identified noise coefficients are Q (quantification noise), $\mathrm{N}$ (angle random walk), B (bias instability), $\mathrm{K}$

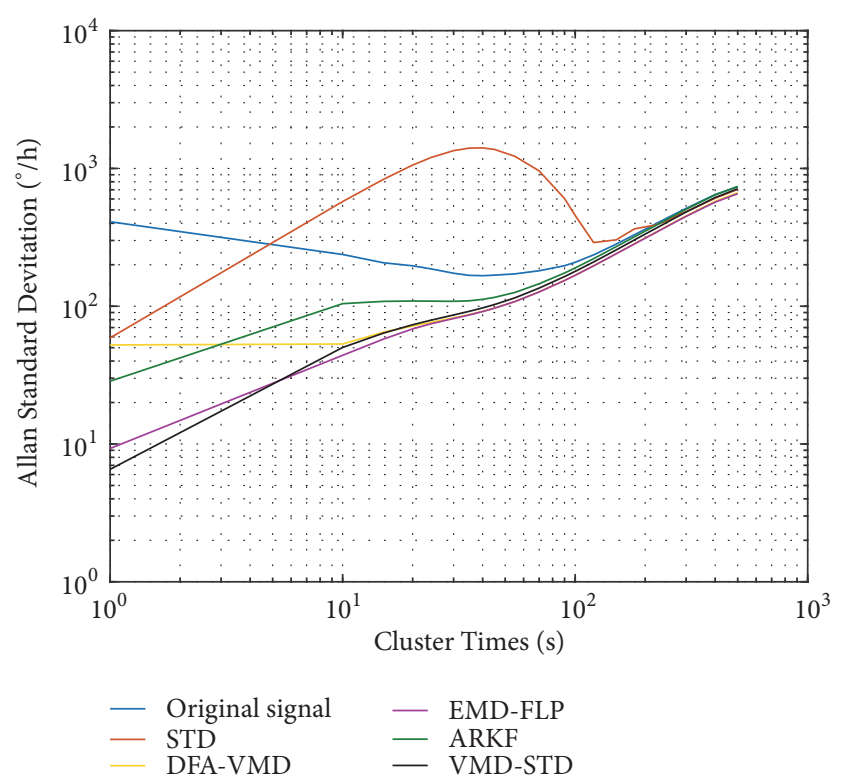

FIGURE 11: Allan variance analysis of gyro's output after compensation.

(rate random walk), and $\mathrm{R}$ (angular rate ramp), respectively, where, $\mathrm{N}$ and $\mathrm{B}$ are the most important parameters to characterize the noise performance of gyroscope. Simply speaking, $\mathrm{N}$ stands for the white noise and $\mathrm{B}$ means the $1 / \mathrm{f}$ noise or other noises induced by environment. From Figure 11 and Table 6 we can see that the $\mathrm{N}$ is $6.84^{\circ} / \mathrm{h}^{1 / 2}$ to $0.10^{\circ} / \mathrm{h}^{1 / 2}$, and the $\mathrm{B}$ is reduced from $6.84^{\circ} / \mathrm{h}$ to $0.10^{\circ} / \mathrm{h}$ after denoising by the proposed VMD-STD algorithm, which performs better than other denoising algorithms.

4.2. Other Discussions. In order to demonstrate the effectiveness of the proposed VMD-STD denoising algorithm, dynamic (step rotation) and bandwidth tests are carried out. Figure 12 is the dynamic test result. The dynamic test is carried 
TABLE 6: Comparison of random noise coefficients by Allan variance analysis.

\begin{tabular}{lccccc}
\hline & $\mathrm{Q}(\mu \mathrm{rad})$ & $\mathrm{N}\left({ }^{\circ} / \mathrm{h}^{1 / 2}\right)$ & $\mathrm{B}\left({ }^{\circ} / \mathrm{h}\right)$ & $\mathrm{K}\left({ }^{\circ} / \mathrm{h}^{3 / 2}\right)$ & $\mathrm{R}\left({ }^{\circ} / \mathrm{h}^{2}\right)$ \\
\hline Original & 103.94 & 6.84 & 166.60 & 126.65 & 189.24 \\
STD & 54.87 & 0.98 & 58.94 & 96.89 & 188.90 \\
DFA-VMD & 48.90 & 0.87 & 52.34 & 85.67 & 189.18 \\
EMD-FLP & 8.54 & 0.15 & 9.28 & 79.20 & 187.47 \\
ARKF & 13.11 & 0.47 & 28.57 & 61.72 & 187.33 \\
VMD-STD & 3.65 & 0.10 & 6.55 & 60.88 \\
\hline
\end{tabular}

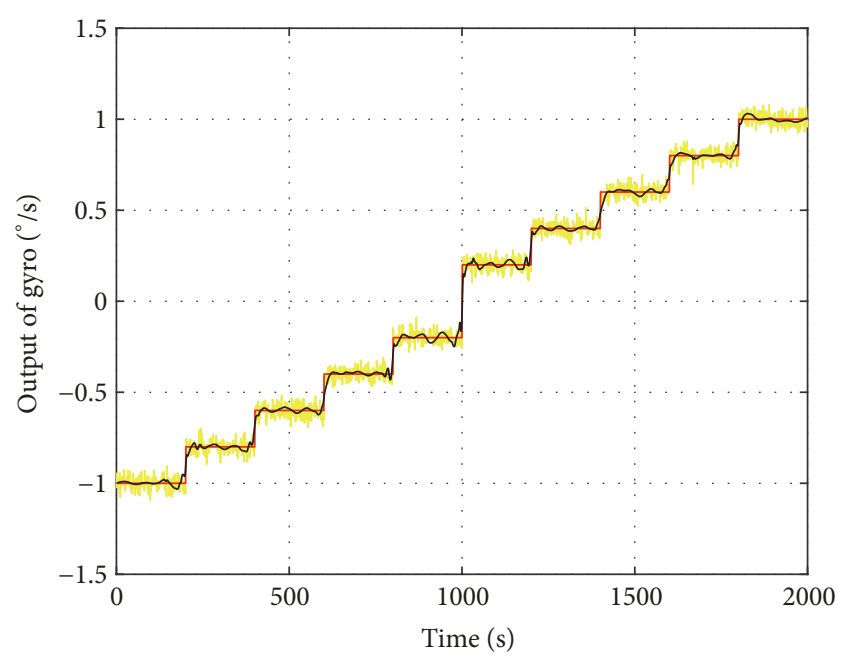

Gyroscope output

- Rotation rate

— De-noising result

FIGURE 12: Dynamic test results.

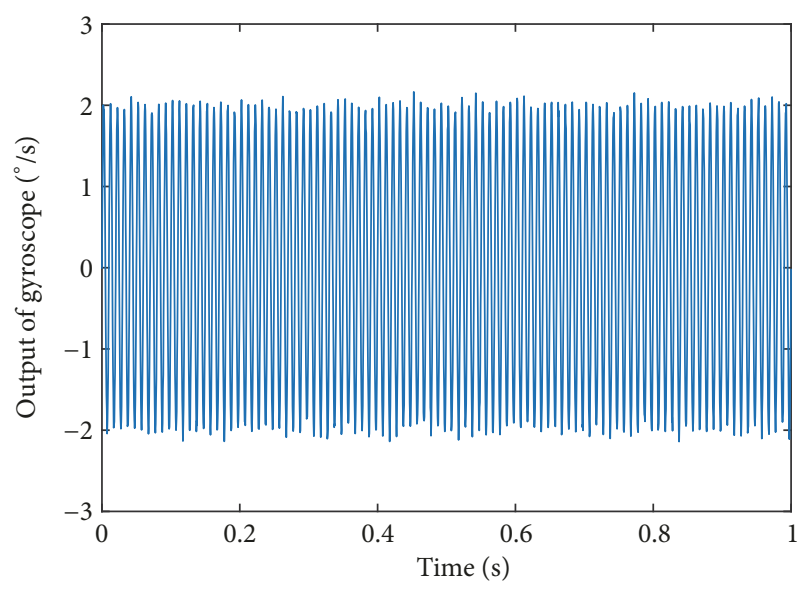

Figure 13: Frequency band of sample signal.

out on rotation plat under constant temperature $\left(25^{\circ} \mathrm{C}\right)$. It can be concluded that the noises are eliminated, and the useful signals are preserved effectively.

Figure 13 is the bandwidth test result. The bandwidth of our gyroscope is $100 \mathrm{~Hz}$. The input angular rate signal is a sine signal (frequency is $100 \mathrm{~Hz}$ ) with $2^{\circ} / \mathrm{s}$ amplitude

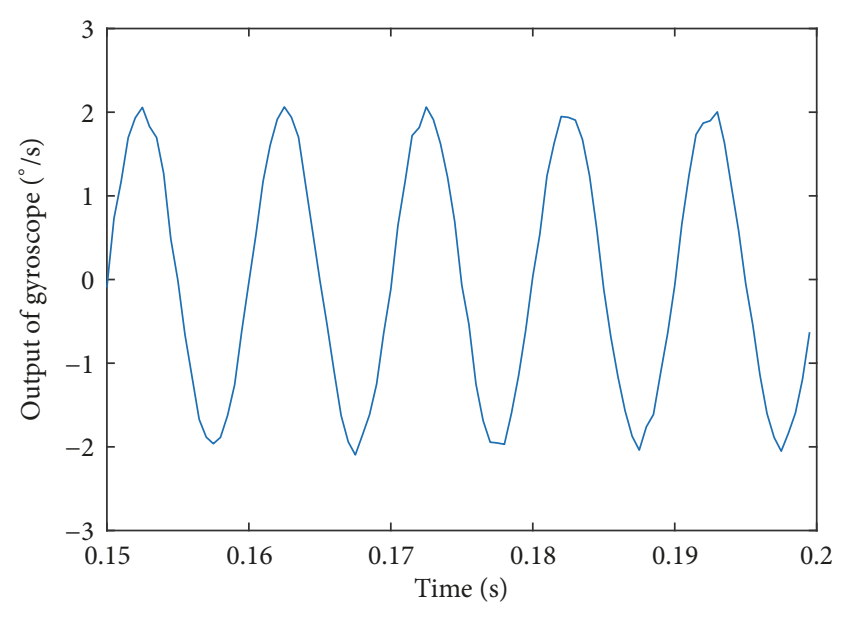

Figure 14: Denosing result.

which is employed for verification. From Figures 13 and 14 we can see that the amplitude of gyroscope output signal keeps $2 \%$, and the curve is smooth which indicates that the noise component is decreased obviously. And the results show that the bandwidth is not reduced by the proposed VMD-STD denoising algorithm.

\section{Conclusions}

To minimize the random noise of MEMS gyroscope, a VMD-STD denoising algorithm is proposed. The main contributions of this paper include the following: firstly, a novel denoising method, named as VMD-STD algorithm, is studied for gyroscope signal processing; secondly the signal delay of STD is solved by combining VMD and STD together; thirdly a VMD-based denoising method is given which makes VMD-based denoising method expanded. The proposed VMD-STD algorithm is verified by the collected MEMS gyroscope data, and the experimental results show that the best denoising result is obtained by using VMDSTD compared to other advanced denoising algorithms, and the temperature drift of MEMS gyroscope can be extracted without signal delay. Additionally, it can be concluded by stationary simulated data and nonstationary experimental output that the proposed denoising algorithm is effective for both stationary and nonstationary signals. 


\section{Data Availability}

The [Original.mat] data used to support the findings of this study are included within the supplementary information file.

\section{Conflicts of Interest}

The authors declare that there are no conflicts of interest regarding the publication of this paper.

\section{Authors' Contributions}

Xi Zhang and Huiliang Cao contributed equally to this paper.

\section{Acknowledgments}

This work was supported in part by the National Natural Science Foundation of China $(61603353,51705477)$, the PreResearch Field Foundation (6140518010201), the Scientific and Technology Innovation Programs of Higher Education Institutions in Shanxi (201802084), the Program for the Top Young Academic Leaders of Higher Learning Institutions of Shanxi, the Young Academic Leaders Foundation in North University of China, Science Foundation of North University of China (XJJ201822), the Fund for Shanxi "1331 Project" Key Subjects Construction, and the Shanxi Province Outstand Researcher (2016M180018).

\section{Supplementary Materials}

The original data are collected from gyroscope with temperature changing from $+10^{\circ} \mathrm{C}$ to $-10^{\circ} \mathrm{C}$ to $+10^{\circ} \mathrm{C}$, where the temperature change rate is less than $1^{\circ} \mathrm{C} / \mathrm{min}$. (Supplementary Materials)

\section{References}

[1] H. Cao, Y. Zhang, Z. Han et al., "Pole-zero-temperature compensation circuit design and experiment for dual-mass mems gyroscope bandwidth expansion," IEEE/ASME Transactions on Mechatronics, 2019.

[2] H. L. Cao, Y. Zhang, C. Shen et al., "Temperature energy influence compensation for MEMS vibration gyroscope based on RBF NN-GA-KF method," Shock and Vibration, vol. 2018, Article ID 2830686, 10 pages, 2018.

[3] S. Chong, S. Rui, L. Jie et al., "Temperature drift modeling of MEMS gyroscope based on genetic-Elman neural network," Mechanical Systems and Signal Processing, vol. 72-73, pp. 897905, 2016.

[4] X. Xu, X. S. Xu, Y. Q. Yao et al., "Optimization-based alignment for inertial navigation systems: theory and algorithm," Review of Scientific Instruments, vol. 88, Article ID 035001, 2017.

[5] X. Guo, J. Tang, J. Li, C. Wang, C. Shen, and J. Liu, "Determine turntable coordinate system considering its non-orthogonality," Review of Scientific Instruments, vol. 90, no. 3, Article ID 033704, 2019.

[6] Q. Zeng, S. Gu, J. Liu et al., "A gyro signal characteristics analysis method based on empirical mode decomposition," Journal of Sensors, vol. 2016, Article ID 3613878, 7 pages, 2016.
[7] C. Shen, J. Yang, J. Tang, J. Liu, and H. Cao, "Note: Parallel processing algorithm of temperature and noise error for microelectro-mechanical system gyroscope based on variational mode decomposition and augmented nonlinear differentiator," Review of Scientific Instruments, vol. 89, no. 7, Article ID 076107, 2018.

[8] Z. Wang, J. Wang, and W. Du, "Research on fault diagnosis of gearbox with improved variational mode decomposition," Sensors, vol. 18, no. 10, Article ID 3510, 2018.

[9] R. Peesapati, S. L. Sabat, K. P. Karthik, J. Nayak, and N. Giribabu, "Efficient hybrid Kalman filter for denoising fiber optic gyroscope signal," Optik - International Journal for Light and Electron Optics, vol. 124, no. 20, pp. 4549-4556, 2013.

[10] R. Peesapati, S. L. Sabat, K. P. Karthik, M. Narasimhappa, N. Giribabu, and J. Nayak, "FPGA-based embedded platform for fiber optic gyroscope signal denoising," International Journal of Circuit Theory and Applications, vol. 42, no. 7, pp. 744-757, 2014.

[11] M. Narasimhappa, S. L. Sabat, R. Peesapati et al., "Fiber-optic gyroscope signal denoising using an adaptive robust kalman filter," IEEE Sensors Journal, vol. 16, no. 10, pp. 3711-3718, 2016.

[12] M. Narasimhappa, S. L. Sabat, and J. Nayak, "Adaptive sampling strong tracking scaled unscented Kalman filter for denoising the fiber optic gyroscope drift signal. IET Science," Measurement \& Technology, vol. 9, no. 3, pp. 241-249, 2015.

[13] H. Guo, Q. Zhu, J. Tang et al., "A temperature and humidity synchronization detection method based on microwave coupledresonator," Sensors and Actuators B: Chemical, vol. 261, pp. 434440, 2018.

[14] J. Cheng, J. Fang, W. Wu, and J. Li, “Temperature drift modeling and compensation of RLG based on PSO tuning SVM," Measurement, vol. 55, pp. 246-254, 2014.

[15] Y. Wu, C. Shen, H. Cao et al., "Improved morphological filter based on variational mode decomposition for MEMS gyroscope de-noising," Micromachines, vol. 9, no. 5, p. 246, 2018.

[16] C. Shen, H. Cao, J. Li et al., "Hybrid de-noising approach for fiber optic gyroscopes combing improved empirical mode decomposition and forward linear prediction algorithms," Review of Scientific Instruments, vol. 87, no. 3, pp. 1-8, 2016.

[17] Z. Kou, J. Liu, H. Cao, Y. Shi, J. Ren, and Y. Zhang, "A novel MEMS S-springs vibrating ring gyroscope with atmosphere package," AIP Advances, vol. 7, no. 12, Article ID 125301, 2017.

[18] X. Shao and H. Wang, "Back-stepping robust trajectory linearization control for hypersonic reentry vehicle via novel tracking differentiator," Journal of The Franklin Institute, vol. 353, no. 9, pp. 1957-1984, 2016.

[19] H. Guo, Y. Chen, D. Wu et al., "Plasmon-enhanced sensitivity of spin-based sensors based on a diamond ensemble of nitrogen vacancy color centers," Optics Expresss, vol. 42, no. 3, pp. 403406, 2017.

[20] K. Dragomiretskiy and D. Zosso, "Variational mode decomposition," IEEE Transactions on Signal Processing, vol. 62, no. 3, pp. 531-544, 2014.

[21] X. Zhang, H. Cao, X. Shao, J. Liu, and C. Shen, "FOG denoising algorithm based on augmented nonlinear differentiator and singular spectrum analysis," Applied Sciences, vol. 8, no. 10, Article ID 1710, 2018.

[22] Z. Kou, J. Liu, H. Cao et al., "Investigation, modeling, and experiment of an MEMS S-springs vibrating ring gyroscope," Journal of Micro/Nanolithography, MEMS, and MOEMS, vol. 17, no. 01, Article ID 015001, 2018. 


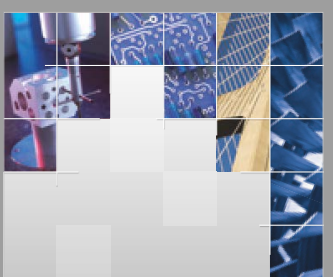

\section{Enfincering}
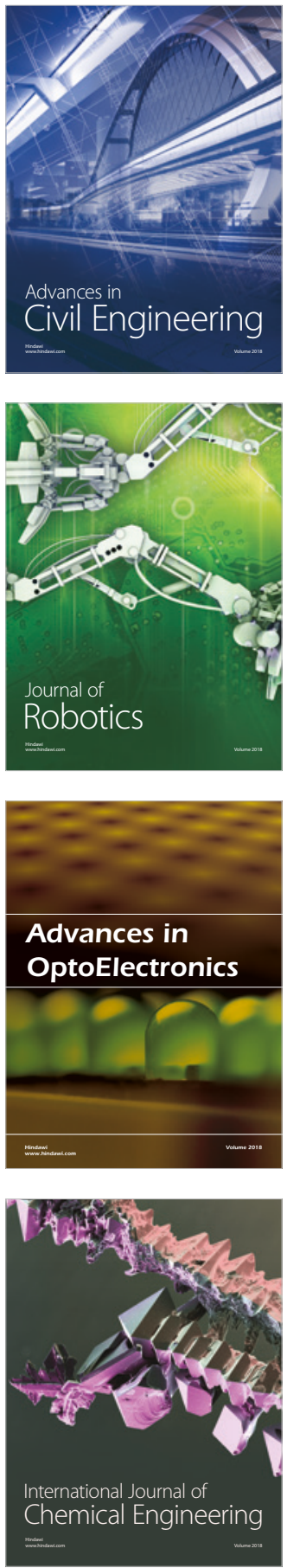

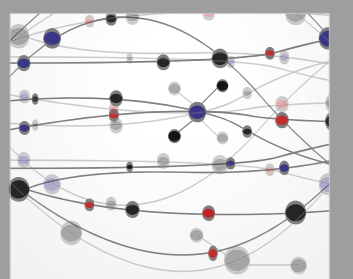

\section{Rotating \\ Machinery}

The Scientific World Journal

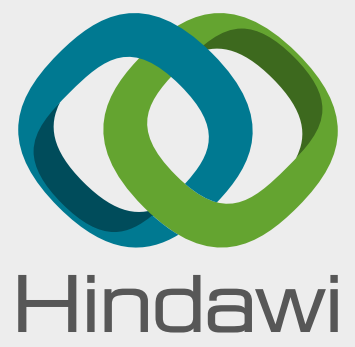

Submit your manuscripts at

www.hindawi.com
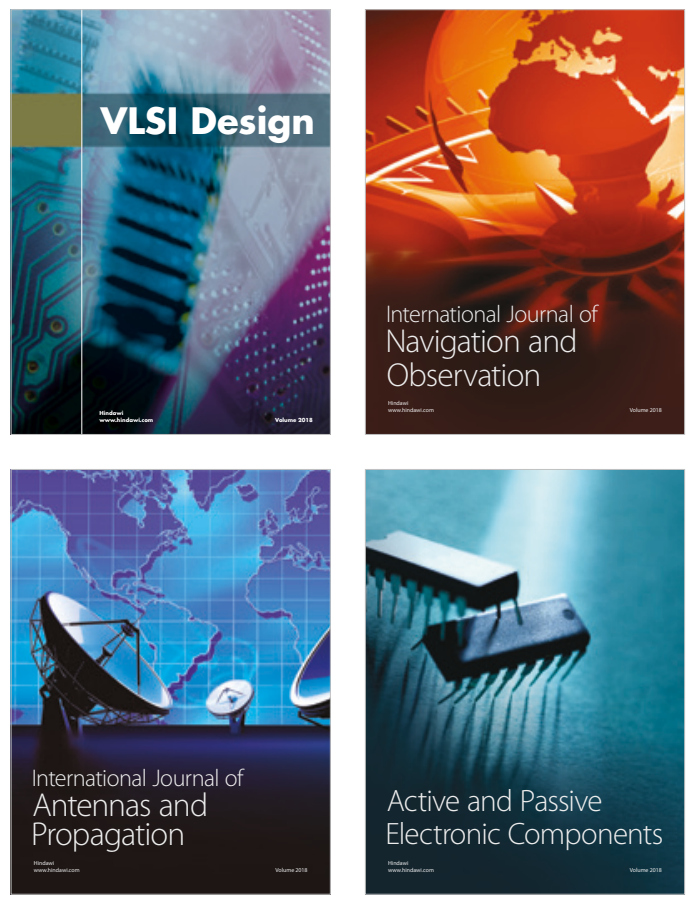
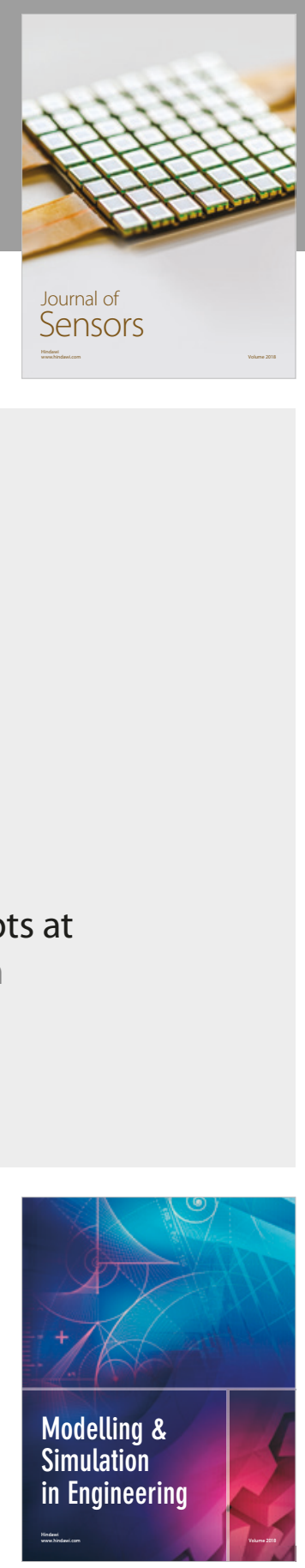

\section{Advances \\ Multimedia}
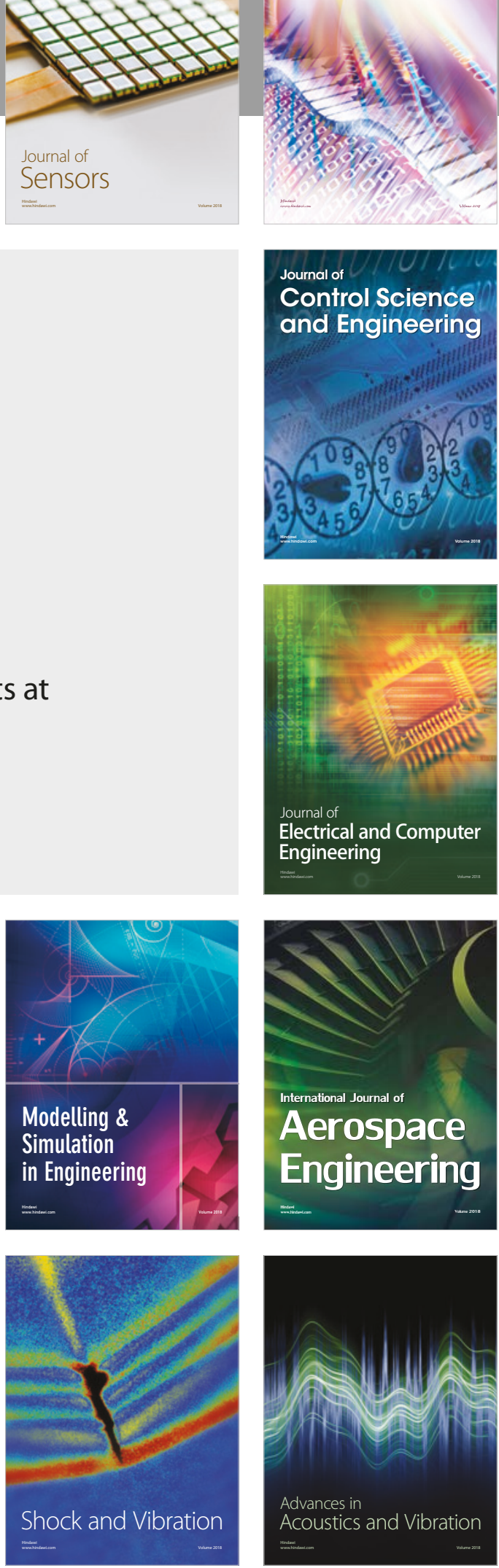